\section{Impact of hypertensive emergency and rare complement variants on the presentation and outcome of atypical hemolytic uremic syndrome}

\author{
Khalil El Karoui,, ${ }^{1}$ Idris Boudhabhay, ${ }^{1}$ Florent Petitprez, ${ }^{2}$ Paula Vieira-Martins, ${ }^{3}$ \\ Fadi Fakhouri, ${ }^{4}$ Julien Zuber, ${ }^{5}$ Florence Aulagnon, ${ }^{5}$ Marie Matignon, ${ }^{1}$ \\ Eric Rondeau, ${ }^{6}$ Laurent Mesnard, ${ }^{6}$ Jean-Michel Halimi ${ }^{7}$ and \\ Véronique Frémeaux-Bacchi ${ }^{3,8}$
}

${ }^{1}$ Service de Néphrologie et Transplantation Rénale, Hôpital Henri Mondor, Assistance Publique-Hôpitaux de Paris, INSERM U955, Créteil; '2INSERM, UMR_S 1138, Centre de Recherche des Cordeliers, F-75006, Paris; ${ }^{3}$ Assistance Publique-Hôpitaux de Paris, Laboratoire d'Immunologie, Hôpital Européen Georges Pompidou, Paris; ${ }^{4}$ Centre de Recherche en Transplantation et Immunologie UMR 1064, INSERM, Université de Nantes and Department of Nephrology and immunology, Centre Hospitalier Universitaire de Nantes, Nantes; ${ }^{5}$ Service de Néphrologie et Transplantation Rénale, Hôpital Necker Enfant malades, Assistance Publique-Hôpitaux de Paris, Paris; ${ }^{\circ}$ Intensive Care and Renal Transplant Unit, Assistance Publique-Hôpitaux de Paris, Centre Hospitalier Universitaire de Tenon and Inserm UMR S 1155, Sorbonne University, Paris; ${ }^{7}$ Department of Nephrology and Clinical immunology, Centre Hospitalier Universitaire de Tours and EA4245, François

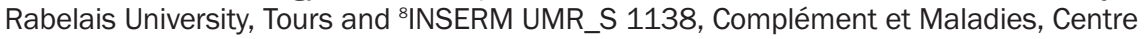
de Recherche des Cordeliers, Paris, France

\section{ABSTRACT}

A typical hemolytic uremic syndrome (aHUS) is a prototypic thrombotic microangiopathy attributable to complement dysregulation. Hypertensive emergency, characterized by elevation of systolic (>180 $\mathrm{mmHg}$ ) or diastolic (>120 $\mathrm{mmHg}$ ) blood pressure together with endorgan damage, can cause thrombotic microangiopathy which may mimic aHUS. We retrospectively evaluated the clinical, biological and complement genetic characteristics of 76 and 61 aHUS patients with and without hypertensive emergency, respectively. Patients with hypertensive emergencyaHUS were more frequently males, with neurological involvement, and a slightly higher hemoglobin level. At least one rare complement variant was identified in $51.3 \%$ (39/76) and $67 \%$ (41/61) patients with or without hypertensive emergency, respectively $(P=0.06)$. In both groups, renal prognosis was severe with $23 \%$ and $40 \%$ of patients reaching end-stage renal disease after a 5 -year follow-up ( $P=0.1)$. The 5 -year renal survival was $77 \%$ in patients without hypertensive emergency or a complement variant, and below $25 \%$ in the three groups of patients with hypertensive emergency and/or a complement variant $(P=0.02)$. Among patients without hypertensive emergency, the 5 -year renal survival was $100 \%$ vs. $40 \%$ in those treated or not with eculizumab, respectively $(P<0.001)$. Conversely, the 5 -year renal survival of patients with hypertensive emergency was $46 \%$ vs. $23 \%$ in those treated or not with eculizumab, respectively $(P=0.18)$. In conclusion, information on the presence or absence of hypertensive emergency and rare complement variants is essential to stratify the long-term renal prognosis of patients with aHUS.

\section{Introduction}

Hemolytic uremic syndrome (HUS) is a thrombotic microangiopathy affecting predominantly the kidney and encompasses a heterogeneous group of disorders, including Shiga toxin producing E. coli-associated HUS (typical HUS), secondary HUS (related to co-existing condition such as malignancy, drugs or autoimmune diseases), and atypical HUS (aHUS). ${ }^{1-3}$ The classification of HUS has evolved with the identification of the mechanisms of endothelial injury. Among patients pre-
Ferrata Storti Foundation
Haematologica 2019
Volume 104(12):2501-2511

\section{Correspondence:}

KHALIL EL KAROUI

khalil.el-karoui@aphp.fr

VÉRONIQUE FRÉMEAUX-BACCHI

veronique.fremeaux-bacchi@aphp.fr

Received: January 21, 2019

Accepted: March 18, 2019.

Pre-published: March 19, 2019.

doi:10.3324/haematol.2019.216903

Check the online version for the most updated information on this article, online supplements, and information on authorship \& disclosures: www.haematologica.org/content/104/12/2501

(C)2019 Ferrata Storti Foundation

Material published in Haematologica is covered by copyright. All rights are reserved to the Ferrata Storti Foundation. Use of published material is allowed under the following terms and conditions:

https://creativecommons.org/licenses/by-nc/4.0/legalcode. Copies of published material are allowed for personal or internal use. Sharing published material for non-commercial purposes is subject to the following conditions: https://creativecommons.org/licenses/by-nc/4.0/legalcode, sect. 3. Reproducing and sharing published material for commercial purposes is not allowed without permission in writing from the publisher. 
senting with aHUS, complement-mediated aHUS is prototypical of disease and occurs as a consequence of hereditary or acquired complement abnormalities. Eculizumab, a monoclonal anti-C5 antibody that blocks the formation of C5b-9 complexes on the surface of endothelial cells, has revolutionized the care of patients with aHUS. ${ }^{4}$ However, in $30-40 \%$ aHUS patients the cause is illdefined and the role of additional genetic or environmental factors remains debatable. ${ }^{1}$

A major complication of HUS is high blood pressure flares related to renal microvascular thromboses and activation of the renin-angiotensin system. Recently, a new concept termed hypertensive emergency (HE) has been introduced to better characterize the syndrome of acute onset high blood pressure with end-organ damage. ${ }^{5,6} \mathrm{HE}$ is defined by severe elevation of systolic or diastolic blood pressure (>180 mmHg or $120 \mathrm{mmHg}$, respectively), associated with progressive organ dysfunction, such as neurological changes, left ventricular failure, or aortic dissection. HE-associated kidney involvement includes renal failure with mechanical hemolytic anemia mimicking aHUS flares. ${ }^{1,3,5-8}$ This latter situation raises the issue of whether primary HUS is complicated by secondary HE, or primary HE leads to secondary HUS.

Whether HE-associated HUS is a complement-mediated disease remains debated. The percentage of patients with $\mathrm{HE}$ is rarely reported in aHUS cohorts. ${ }^{4,9}$ In a recent study, no pathogenic or likely pathogenic variants were identified in 100 non-elderly patients presenting with severe hypertension, renal failure and a kidney biopsy showing arteriolar thrombotic microangiopathy. ${ }^{10}$ However, in a large study of 273 patients with aHUS, 14 patients $(5 \%)$ also had hypertensive flares, with mutations affecting the regulation of the alternative complement pathway in $2 / 14 .{ }^{11}$ Moreover, a recent case series reported that $8 / 17$ patients with 'hypertension-associated thrombotic microangiopathy' carried a pathogenic variant in the genes for complement factor $\mathrm{H}(\mathrm{CFH})$, complement factor I (CFI), membrane cofactor protein $(M C P)$ or complement component 3 (C3). ${ }^{12}$

In the current study, in a large French cohort we analyzed the clinical, biological and genetic characteristics of patients with aHUS and HE at onset (HE-aHUS), and compared the findings with those of aHUS patients without HE (noHE-aHUS). We showed that patients with HEaHUS have specific clinico-biological characteristics compared to those of patients without HE.

\section{Methods}

\section{Patients}

HUS was defined by renal involvement (acute renal failure or proteinuria) with mechanical hemolytic anemia (including low hemoglobin, elevated lactate dehydrogenase, and/or the presence of schistocytes) and/or thrombocytopenia. Between 2000 and 2016, we screened 405 patients with adult-onset HUS and ADAMTS-13 activity $>15 \%$ for complement function and genetic abnormalities. To retrospectively develop this study and identify patients with or without HE and HUS, the patients' medical records were reviewed and relevant clinical and biological data were collected. Patients without blood pressure data were excluded from this retrospective study.

For this study, the exclusion criteria were: (i) HUS with coexisting diseases (such as infection-induced HUS, monoclonal gammopathy, solid-organ transplantation, hematopoietic stem cell transplantation or malignancy) or (ii) lack of reliable blood pressure data. We adopted the term aHUS to define HUS without co-existing diseases.

HE was defined according to the 2013 European Society of Hypertension/European Society of Cardiology guidelines (systolic blood pressure $\geq 180 \mathrm{mmHg}$ and/or diastolic blood pressure $>120 \mathrm{mmHg}$ ), together with end-organ damage, such as renal, neurological, cardiac or ophthalmological involvement. ${ }^{6}$ Blood pressure was measured repeatedly during the initial diagnosis. Neurological involvement included acute onset severe headache, confusion, seizures, cerebral infarction/hematoma, and cerebral magnetic resonance images compatible with posterior reversible encephalopathy syndrome (PRES). Cardiac involvement included acute left or right ventricular dysfunction and cardiac arrythmia.

HE-aHUS and noHE-aHUS were defined as aHUS with or without HE, respectively. This study includes patients previously reported by van der Born et al. ${ }^{8}$ We retrospectively found that ten patients published as having aHUS in 2013 had HE-aHUS at onset. ${ }^{9}$

\section{Complement analyses}

Complement evaluation and genetic analyses were performed as part of the usual work-up of patients diagnosed with aHUS. Plasma concentrations of C3, C4, factor B, factor $\mathrm{H}$ and factor I, and MCP expression on granulocytes were quantified as previously described. ${ }^{9}$ All coding sequences of the CFH, CFI, MCP, C3, complement factor $\mathrm{B}(C F B)$ and thrombomodulin (THBD) genes were analyzed by direct sequencing as previously described or by next-generation sequencing. ${ }^{13}$ In our study, we defined a variant as rare when its minor allele frequency was below $1 \%$ in the general population. Among these rare variants, we named as pathogenic those for which the genetic change affects protein function (well-established in vitro functional studies supportive of a damaging effect on the gene product), and/or the genetic change was found in a disease-related functional domain, and/or affects protein expression (nonsense, frameshift, canonical +/- one or two splice sites variants, well-demonstrated lack of in vitro synthesis, or quantitative deficiency in the patient's plasma) (definitions adapted from Richards et al. ${ }^{14}$ and Goodship et al. ${ }^{15}$ ). The other variants were classified as variants of uncertain significance.

All patients gave informed consent for genetic analyses. The study was approved by the ethics committee of the French national clinical research projects authority number AOM08198). DNA samples available from 80 healthy blood donors were also sequenced for the same genes.

\section{Statistical analyses}

Data are presented as percentages or mean \pm standard deviation. The Fisher exact test was used to compare qualitative data. Renal survival was analyzed with Kaplan-Meier estimates and the log-rank test or by univariate and multivariate Cox proportional hazards regression when indicated. A $P$ value $<0.05$ was considered statistically significant.

\section{Results}

\section{Clinical and biological characteristics of atypical hemolytic uremic syndrome patients with or without hypertensive emergency}

Of 405 HUS patients, 142 were excluded because of coexisting disease, and 126 had no blood pressure data. Thus, 137 patients with aHUS were enrolled in this study. 
Of these, $76(54 \%)$ had concomitant HE (HE-aHUS), and 61 did not have HE (noHE-aHUS) (Figure 1). A total of 7/44 noHE-aHUS females, and 1/32 HE-aHUS females were diagnosed after pregnancy. Eculizumab was used in 13/76 (17\%) HE-aHUS and 17/61 (28\%) noHE-aHUS patients. The median follow-up was 39.9 months, and 57 patients presented with definitive end-stage renal disease at onset. Follow-up was not available for two patients with HE-aHUS.

The patients' clinical and biological characteristics are presented in Table 1 . The male/female ratio of the 76 patients with HE-aHUS was 44/32 (male 58\%). The patients' mean age was 37 years, and their mean systolic/diastolic blood pressure was $214 / 128 \mathrm{mmHg}$. The mean hemoglobin concentration was $8.5 \mathrm{~g} / \mathrm{dL}$ and thrombocytopenia was profound [mean $104 \times 10^{\%} / \mathrm{L}$; platelet count $<100 \times 10^{9} / \mathrm{L}$ in $42 \%$ (32 patients)]. Acute kidney injury was severe with $81 \%$ patients requiring dialysis at onset. Twelve of the 76 patients $(16 \%)$ presented with a diagnosis of long-lasting high blood pressure or left ventricular hypertrophy. Kidney biopsy, performed in $24 \mathrm{HE}-$ aHUS patients $(32 \%)$, showed typical features of thrombotic microangiopathy with arteriolar thromboses, except in one patient with only glomerular retraction suggestive of glomerular ischemia. The patients with $\mathrm{HE}-$ aHUS had a severe prognosis, since 1 -year and 5 -year renal survival rates were $36 \%$ and $23 \%$, respectively, in patients not treated with eculizumab (Figure 2).

Compared to patients with noHE-aHUS (Table 1), HEaHUS patients were more frequently males $(58 \%$ vs. $28 \%, P<0.01)$ with a significantly higher frequency of neurological involvement and higher hemoglobin levels (8.5 vs. $7.5 \mathrm{~g} / \mathrm{dL}, P=0.01$ ). No significant difference was observed in age, cardiac involvement, platelet count, presence of elevated lactate dehydrogenase, schistocyte frequency or dialysis requirement at onset between the two groups (Table 1).

Four deaths occurred among the $76 \mathrm{HE}-\mathrm{aHUS}$ patients during follow-up, whereas there were five deaths among the 61 noHE-aHUS patients $(5.2 \%$ vs. $8.2 \%$ respectively, $P=0.5)$. The deaths were related to sepsis ( 3 patients), a cardiac etiology (2 patients), cancer (1 patient), cerebral hematoma (1 patient), and to an unknown cause (2 patients). Five of these nine patients already had endstage renal disease before death. Without eculizumab treatment, the prognosis of HE-aHUS $(n=61)$ and noHEaHUS $(n=44)$ patients was similar, since 1 -year and 5 -year renal survival rates were $36 \%$ vs. $45 \%$, and $23 \%$ vs. $40 \%$ in the HE-aHUS and noHE-aHUS patients, respectively $(P=0.1)$ (Figure 2). However, in the whole cohort including patients treated with eculizumab, those with $\mathrm{HE}$ aHUS had a worse renal prognosis than those with noHE-

\section{French HUS registry}

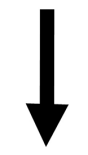

\section{5 patients were screened for rare genetic variants in complement}

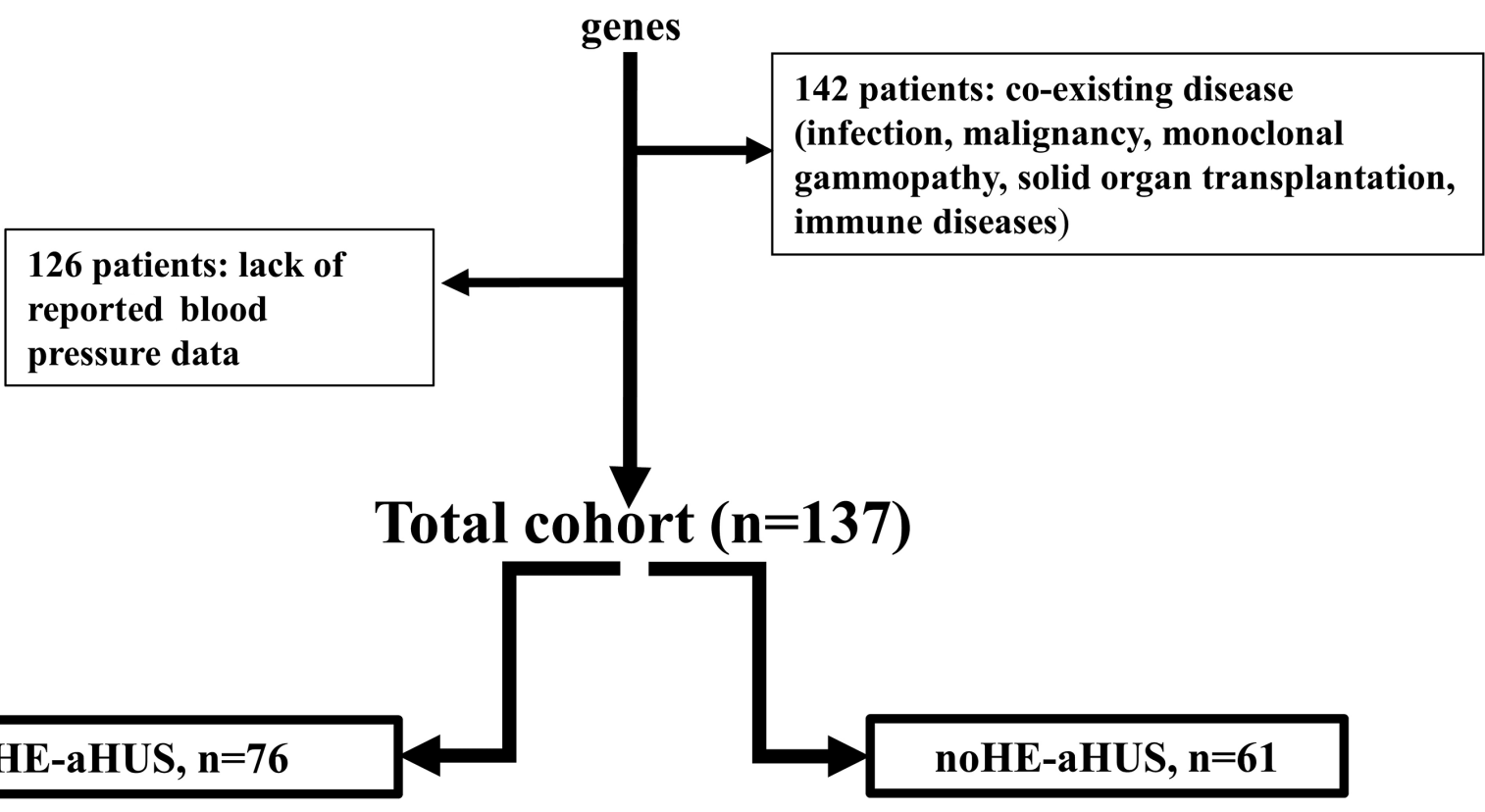

Figure 1. Study flow chart. Flow chart for the inclusion criteria of patients within the adult population of the French HUS registry screened for genetic abnormalities $(n=405)$. A total of 137 patients were eligible for enrollment in the study. HUS: hemolytic uremic syndrome; HE: hypertensive emergency; aHUS: atypical hemolytic uremic syndrome. 
aHUS (1-year and 5-year renal survival rates $42 \%$ vs. $62 \%$ and $27 \%$ vs. $56 \%$, respectively, $P=0.002$ ) (Online Supplementary Figure S1). Similar results were obtained when analyzing death-censored renal survival (Online Supplementary Figure S2A-F).

\section{Complement evaluation}

Results of the complement work-up in HE-aHUS and noHE-aHUS patients are presented in Tables 1 and 2. Low C3 levels were reported less frequently in patients with HE-aHUS than in those with noHE-aHUS (12/76 vs. $19 / 58, P=0.02)$. CFH autoantibodies with homozygous complement factor $\mathrm{H}$-related protein 1 (CFHR1) deletion were detected in 2/76 patients with HE-aHUS (2/76) and 1/61 patients with noHE-aHUS.

At the individual level, a rare variant in the candidate genes was detected in 39/76 (51.3\%) HE-aHUS patients, which was a slightly lower incidence that in noHE-aHUS patients $(41 / 61,67 \%, P=0.06)$, but significantly higher than in healthy donors $(13.7 \%, P<0.0001)$. The distribu- tion of each rare variant did not differ between HE-aHUS and noHE-aHUS patients: $C F H 22 \%$ vs. $34 \%$ ( $P=$ ns), MCP $2.6 \%$ vs. $5 \%$ ( $P=$ ns $), C F I 11.9 \%$ vs. $8.2 \%(P=$ ns $)$, C3 $5.3 \%$ vs. $11.5 \%$ ( $P=\mathrm{ns})$, CFB $1.3 \%$ vs. $3 \%(P=\mathrm{ns})$, THBD $1.3 \%$ vs. $0 \%(P=$ ns $)$ and more than one variant $6.6 \%$ vs. $5 \%$ $(P=\mathrm{ns})$, respectively (Table 2$)$. We found genomic rearrangements involving $C F H$-CFHR1 in four HE-aHUS $(5 \%)$ patients and two noHE-aHUS $(3 \%)$ patients. To investigate the consequences of the rare variants on protein expression and function, we analyzed the variant pathogenicity. Among the 45 rare variants identified in HE-aHUS patients, a total of $30 / 45(66 \%)$ variants were pathogenic, and located in the coding regions of $C F H$ $(\mathrm{n}=16), M C P(\mathrm{n}=2), C F I(\mathrm{n}=9)$ and $C 3(\mathrm{n}=3)$ (Figure 3 and Online Supplementary Tables S1-4).

In HE-aHUS patients, 30/76 (39.4\%) carried at least one pathogenic variant, a frequency lower than that in noHEaHUS patients $(38 / 61,62 \% ; P=0.008)$ but higher than in controls $(2 / 80,2.5 \%$; $P<0.0001)$ (Table 2$)$.

The frequencies of the homozygous at-risk MCP

Table 1. Clinical, biological and genetic characteristics of patients with atypical hemolytic uremic syndrome with or without hypertensive emergency.

\begin{tabular}{lcccc} 
& Whole cohort & HE-aHUS & noHE-aHUS & P-value \\
Number & 137 & 76 & 61 & \\
Male gender, $\mathrm{n}(\%)$ & $61(45)$ & $44(58)$ & $17(28)$ & 0.003 \\
\hline
\end{tabular}

\section{At diagnosis}

\begin{tabular}{|c|c|c|c|c|}
\hline SBP/DBP, mean, mmHg & $190 / 112$ & $214 / 128$ & $154 / 90$ & $<0.0001$ \\
\hline Age, mean (SD), years & $37(12)$ & $37(11)$ & $36(13)$ & 0.2 \\
\hline eGFR at onset, mean (SD), mL/min/1.73 m² & $14(14)$ & $12.9(12)$ & $15.5(17)$ & 0.3609 \\
\hline Hemoglobin, mean (SD), g/dL & $8(1.9)$ & $8.5(2)$ & $7.5(1.5)$ & 0.0112 \\
\hline Platelets, mean (SD), x10/L & $97(5)$ & 104(5.5) & $88(8.4)$ & 0.11 \\
\hline Elevated LDH, n (\%) & $67 / 79(84)$ & $34 / 42(93)$ & $33 / 37(95)$ & 0.4 \\
\hline Presence of schistocytes, n (\%) & 80/96(83) & $41 / 53(77)$ & $39 / 42(92)$ & 0.11 \\
\hline Neurologic impairment, n (\%) & 44/102(43) & $32 / 50(64)$ & $12 / 52(23)$ & $<0.0001$ \\
\hline Cardiac dysfunction, $\mathrm{n}(\%)$ & 22/113(19) & $16 / 61(26)$ & $6 / 52(12)$ & 0.059 \\
\hline Dialysis at onset $\mathrm{n}(\%)$ & 98/126(78) & $57 / 70(81)$ & $41 / 56(73)$ & 0.28 \\
\hline \multicolumn{5}{|l|}{ At last follow up } \\
\hline Death & 9 & 4 & 5 & 0.5 \\
\hline Dialysis n(\%) & 97/126(77) & $48 / 71(81)$ & $40 / 56(71)$ & 0.01 \\
\hline Age at dialysis, mean (SD), years & $37.7(12)$ & $37.3(12)$ & $37.9(12)$ & 0.84 \\
\hline Time until ESRD, mean (SD), m & $21(51)$ & $13(31)$ & $36(75)$ & 0.07 \\
\hline \multicolumn{5}{|l|}{ Complement component assessment } \\
\hline C3 (660 to 1250 mg/L), median (Q1,Q3) & $817(676 ; 941)$ & $841(692 ; 976)$ & $768(655 ; 891)$ & 0.04 \\
\hline Low C3 (<660mg/L) n (\%) & $31 / 134(23)$ & $12 / 76(15.7)$ & $19 / 58(31)$ & 0.02 \\
\hline C4 (93 to $380 \mathrm{mg} / \mathrm{L})$ & $253(206 ; 312)$ & $263(215 ; 314)$ & $246(170 ; 299)$ & 0.05 \\
\hline Low factor $\mathrm{H}(<338 \mathrm{mg} / \mathrm{L}) \mathrm{n}(\%)$ & 24/134 (18) & $11 / 76(14,5)$ & $13 / 58(21.6)$ & 0.2 \\
\hline Low factor I ( $<42 \mathrm{mg} / \mathrm{L})$ n $(\%)$ & $16 / 134(12)$ & $8 / 76(10.5)$ & $7 / 58(11.6)$ & 0.8 \\
\hline Low CD46 (<12 MFI) n (\%) & 4/134 (2.9) & $1 / 76(1.3)$ & $3 / 58(5)$ & 0.2 \\
\hline Positive anti-factor H Ab, n (\%) & $3 / 123(2.4)$ & $2 / 76(2.6)$ & $1 / 47(2)$ & 0.8 \\
\hline Rare variants in complement genes $n(\%)$ & $80(58.4)$ & $39(51.3)$ & $41(67)$ & 0.06 \\
\hline
\end{tabular}

HE: hypertensive emergency; aHUS: atypical hemolytic uremic syndrome; SBP: systolic blood pressure; DBP: diastolic blood pressure; SD: standard deviation; eGFR: estimated glomerular filtration rate; LDH: lactate dehydrogenase; ESRD: end-stage renal disease; Ab: antibodies. 
ggaaac and $C F H$ tgtgt haplotypes were significantly higher in HE-aHUS patients than in controls (MCP ggaac $27 \%$ vs. $6.2 \%$; CFH tgtgt $16 \%$ vs. $3.7 \%$ ) (Table 2 ). Notably, $8 / 74(11 \%)$ HE-aHUS patients were homozygous for both at-risk haplotypes, whereas none of the control population was.

Among HE-aHUS patients, three rare variants were identified in six Afro-Caribbean patients, compared to 37 variants in 70 patients of other ethnic origin, defining a similar frequency of rare complement variants in both ethnic groups (50\% vs. 53\%, respectively). Only one of the six Afro-Caribbean patients was homozygote for the at-risk ApoL1 haplotype, a similar frequency as that in the general Afro-Caribbean population. ${ }^{16}$

\section{Treatment of atypical hemolytic uremic syndrome with or without hypertensive emergency}

All HE-aHUS patients were initially treated with antihypertensive therapy. Plasma infusion or plasma exchange (PLEX) was used in 39/57 HE-aHUS patients vs. $47 / 52$ noHE-aHUS patients (68\% vs. $90 \% ; P=0.009)$. However, no difference was observed in prognosis according to PLEX treatment (Online Supplementary Tables S5 and S6, Online Supplementary Figure S3).

Eculizumab was used in 13/76 (17\%) HE-aHUS patients and $17 / 61(28 \%)$ noHE-aHUS patients. In HEaHUS patients, eculizumab was usually proposed after failure of PLEX and anti-hypertensive therapy (median time between diagnosis and eculizumab, 10 days).
Overall, eculizumab provided a significant benefit in the whole cohort (HE-aHUS and noHE-aHUS patients), with a 5 -year renal survival of $79 \%$ vs. $30 \%$ in patients treated or not with eculizumab, respectively $(P<0.001)$ (Figure 4A, Online Supplementary Table $S 5 A, B)$. Notably,

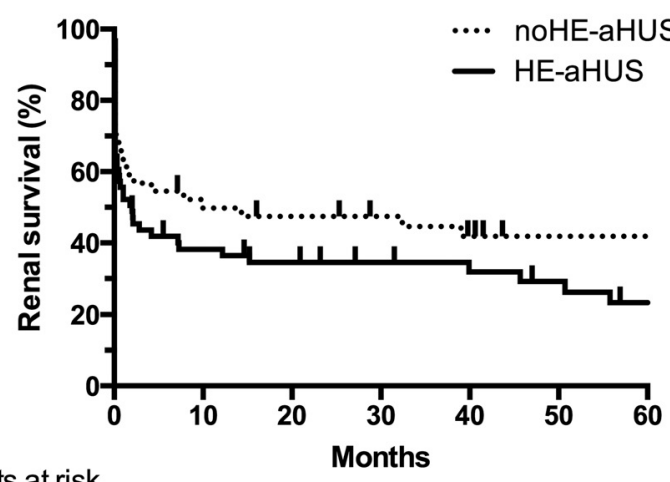

Patients at risk

$\begin{array}{llllllll}\text { noHE-aHUS } & 44 & 22 & 20 & 18 & 14 & 12 & 12 \\ \text { HE-aHUS } & 61 & 22 & 17 & 15 & 13 & 10 & 8\end{array}$

Figure 2. Renal survival in patients with atypical hemolytic uremic syndrome, with or without hypertensive emergency, not treated with eculizumab. Analysis of renal survival without end-stage renal disease or death in patients not treated with eculizumab. Log-rank test, $P=0.1$. Follow-up was not available for two patients with hypertensive emergency and atypical hemolytic uremic syndrome. HE: hypertensive emergency; aHUS: atypical hemolytic uremic syndrome.

Table 2. Genetic characteristics of patients with atypical hemolytic uremic syndrome with or without hypertensive emergency, and of French controls.

\begin{tabular}{|c|c|c|c|c|c|c|}
\hline & $\begin{array}{c}\text { HE- aHUS } \\
n=76\end{array}$ & $\begin{array}{c}\text { noHE-aHUS } \\
n=61\end{array}$ & $\begin{array}{l}\text { French controls } \\
n=80\end{array}$ & $\begin{array}{l}\text { P (HE-aHUS vS. } \\
\text { French controls) }\end{array}$ & $\begin{array}{l}P \text { (noHE-aHUS vs. } \\
\text { French controls) }\end{array}$ & $\begin{array}{c}P \text { (HE-aHUS vs. } \\
\text { no HE-aHUS) }\end{array}$ \\
\hline Individuals with rare variant & $39(51.3)$ & $41(67)$ & $11(13.7)$ & $<0.0001$ & $<0.0001$ & 0.06 \\
\hline Individuals with at least one pathogenic variant & $30(39.4)$ & $38(62)$ & $2(2.5)$ & $<0.0001$ & $<0.0001$ & 0.008 \\
\hline CFH rare variant, $\mathrm{n}(\%)$ & $17(22.4)$ & $21(34.4)$ & $2(2.5)$ & 0.0001 & 0.0003 & 0.12 \\
\hline Pathogenic variant, $\mathrm{n}$ (\% of $\mathrm{CFH}$ rare variants) & $15(88)$ & $20(95)$ & $0(0)$ & & & \\
\hline$M C P$ rare variant, $\mathrm{n}(\%)$ & $2(2.6)$ & $3(5)$ & 0 & 0.2 & 0.07 & 0.6 \\
\hline Pathogenic variant, $\mathrm{n}$ (\% of $M C P$ rare variants) & $2(100)$ & $3(100)$ & $0(0)$ & & & \\
\hline CFI rare variant, $\mathrm{n}(\%)$ & $9(11.9)$ & $5(8.2)$ & $2(2.5)$ & 0.04 & 0.07 & 0.4 \\
\hline Pathogenic variant, $n$ (\% of $C F I$ rare variants) & $7(78)$ & $4(80)$ & $0(0)$ & & & \\
\hline C3 rare variant, $n(\%)$ & $4(5.3)$ & $7(11.5)$ & $4(5)$ & 0.9 & 0.17 & 0.5 \\
\hline Pathogenic variant, $\mathrm{n}$ (\% of $C 3$ rare variants) & $2(50)$ & $6(85)$ & $0(0)$ & & & \\
\hline CFB rare variant, $\mathrm{n}(\%)$ & $1(1.3)$ & $2(3)$ & 0 & 0.5 & 0.2 & 0.6 \\
\hline Pathogenic variant, $\mathrm{n}$ (\% of $C 3$ rare variants) & 0 & $2(100)$ & $0(0)$ & & & \\
\hline THBD rare variant, $\mathrm{n}(\%)$ & $1(1.3)$ & $0(0)$ & $1(1.25)$ & 0.5 & 0.6 & 0.4 \\
\hline Pathogenic variant, $\mathrm{n}$ (\% of $T H B D$ rare variants) & 0 & 0 & $1(100)$ & & & \\
\hline Two rare variants n (\%) & $5(6.6)$ & $3(5)$ & $2(2.5)$ & 0.2 & 0.45 & 0.4 \\
\hline $\begin{array}{l}\text { Patients with at least one Pathogenic variant, } n \\
\text { (\% of patients with } 2 \text { rare variants) }\end{array}$ & $4(80)$ & $3(100)$ & $1(50)$ & & & \\
\hline Anti-CFH antibodies n (\%) & $2(2.6)$ & $1(2)$ & 0 & 0.2 & 0.2 & 0.3 \\
\hline At-risk homozygous CFH tgtgt haplotype n (\%) & $12 / 74(16)$ & $6 / 56(10.7)$ & $3 / 80(3.7)$ & 0.02 & 0.1 & 0.3 \\
\hline At-risk homozygous $M C P$ ggaac haplotype n (\%) & $20 / 74(27)$ & $15 / 50(30)$ & $5 / 80(6,25)$ & 0.002 & 0.001 & 0.9 \\
\hline Both haplotypes n (\%) & $8 / 74(11)$ & $2 / 50(4)$ & 0 & 0.04 & 0.2 & 0.2 \\
\hline
\end{tabular}

HE: hypertensive emergency; aHUS: atypical hemolytic uremic syndrome; n: number of individuals; $C F B$ : complement factor $\mathrm{B}$; $C F H$ : complement factor $\mathrm{H} ; C F F$ : complement factor $\mathrm{I} ; M C P$. membrane cofactor protein; THBD: thrombomodulin. None of the patients carry two rare variants in the DGKE gene. 
eculizumab had a major beneficial effect in noHE-aHUS patients, since their 5-year renal survival was $100 \%$ vs. $40 \%$ in those treated or not with eculizumab, respectively $(P<0.001)$ (Figure 4B). Conversely, in HE-aHUS patients, we did not observe a significant improvement in prognosis with eculizumab (5-year renal survival 46\% vs. $23 \%$ in HE-aHUS patients treated or not with eculizum$\mathrm{ab}$, respectively; $P=0.18$ ) (Figure 4C, Online Supplementary Table S6). The presence of rare complement variants was associated with a trend to improved prognosis after eculizumab treatment, since the 1-year renal survival was $85 \%$ vs. $50 \%$ in HE-aHUS patients with or without rare complement variants, respectively $(P=0.06)$ (Online Supplementary Figure S4).

Multivariate analysis demonstrated that eculizumab treatment and dialysis at onset were independently asso- ciated with renal prognosis in the whole cohort, but not in HE-aHUS patients (Online Supplementary Tables $S 5$ and S6).

\section{Characteristics and outcome according to clinical factors and rare complement variants}

The patients' characteristics were studied according to the presence or absence of $\mathrm{HE}$ and rare complement variants (Table 3A, B). Among HE-aHUS patients, only slight differences in age and platelet count were observed between patients with or without rare complement variants. However, low C3 was significantly more frequent in cases with rare complement variants (11/41 vs. 1/35; $P=0.004)$.

After exclusion of patients treated with eculizumab, a comparison of outcome according to the presence of $\mathrm{HE}$

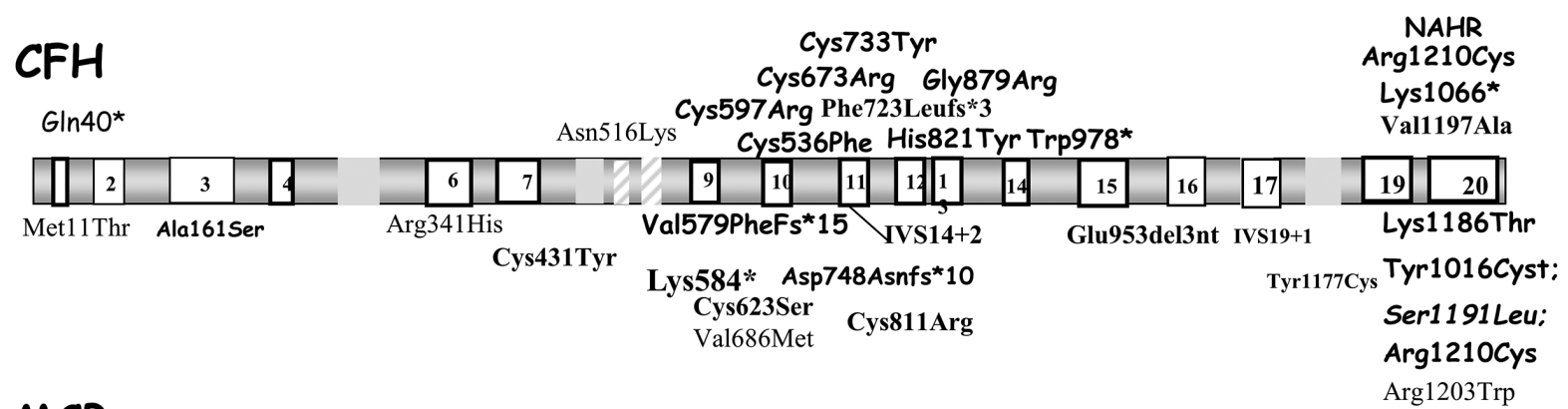

MCP

NAHR
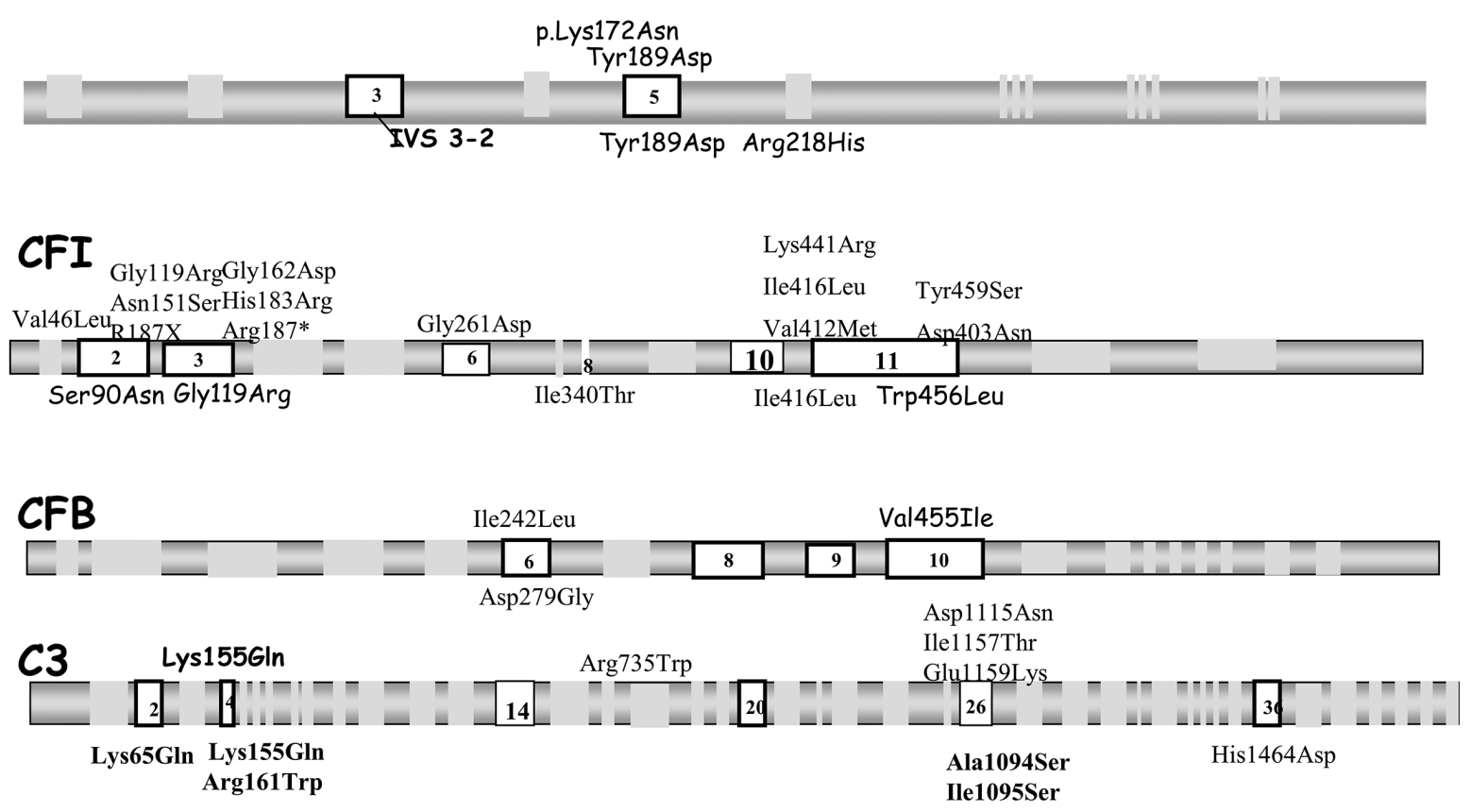

THBD G77S

Figure 3. Distribution of rare complement variants observed in the whole cohort. Rare complement genetic variants found in patients with atypical hemolytic uremic syndrome are presented above and below the schematic gene representation for those with or without hypertensive emergency, respectively. The nucleotide and amino acid numbering refer to the translation start site (A in ATG is +1 ), as recommended by the Human Genome Variation Society. Bold characters indicate mutations identified in two or more unrelated patients, suggesting that they may represent mutational hot spots. $\mathrm{CFH}$ : complement factor $\mathrm{H}$; MCP: membrane cofactor protein; CFI: complement factor I; CFB: complement factor B; C3: complement component 3: THBD: thrombomodulin. 
or a rare complement variant disclosed major differences in renal survival: patients without either HE or complement variants had a significantly better outcome than all other groups (with $\mathrm{HE}$, and/or rare complement variants) (Figure 5A). Five-year renal survival rates were $77 \%$ (no $\mathrm{HE}$ and no complement variant), 22\% (HE without a complement variant), $25 \%$ (complement variant without $\mathrm{HE}$ ) and $23 \%$ (HE with a complement variant) $(P=0.02)$ (Figure 5A). The median serum creatinine and estimated glomerular filtration rate of patients without either $\mathrm{HE}$ or a complement variant at last follow-up were $75 \mu \mathrm{M}$ and $82 \mathrm{~mL} / \mathrm{min} / 1.73 \mathrm{~m}^{2}$, respectively. No difference in outcome was observed according to the type of complement variant (data not shown). Similar results were obtained when analyzing the whole cohort, including patients treated with eculizumab, since 5 -year renal survival rates were $84 \%$ (no $\mathrm{HE}$ and no complement variant), 21\% (HE without a complement variant), 44\% (complement variant without $\mathrm{HE}$ ) and $34 \%$ (HE with a complement variant) $(P=0.001)$ (Figure $5 B)$.

A

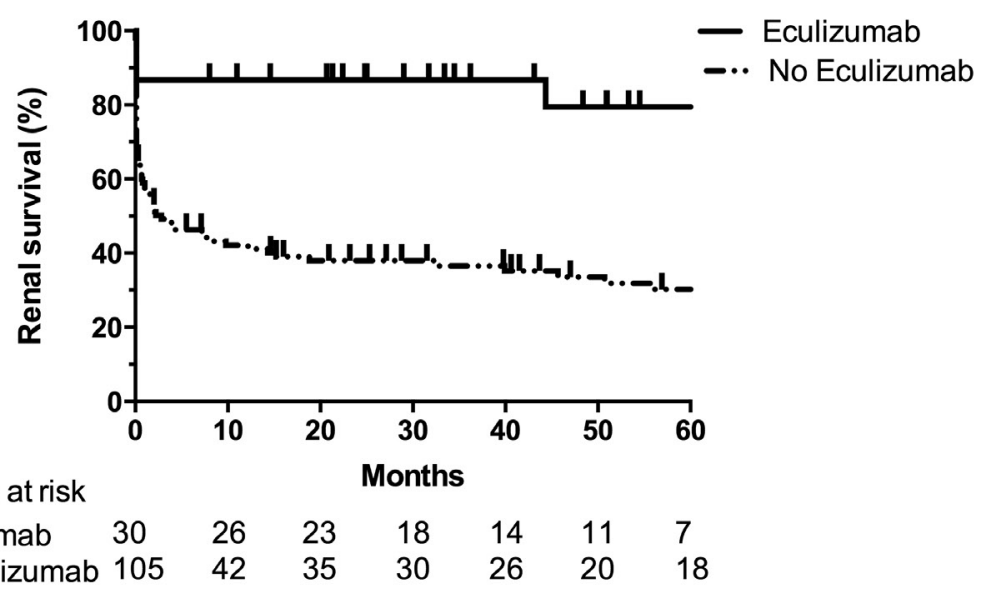

B

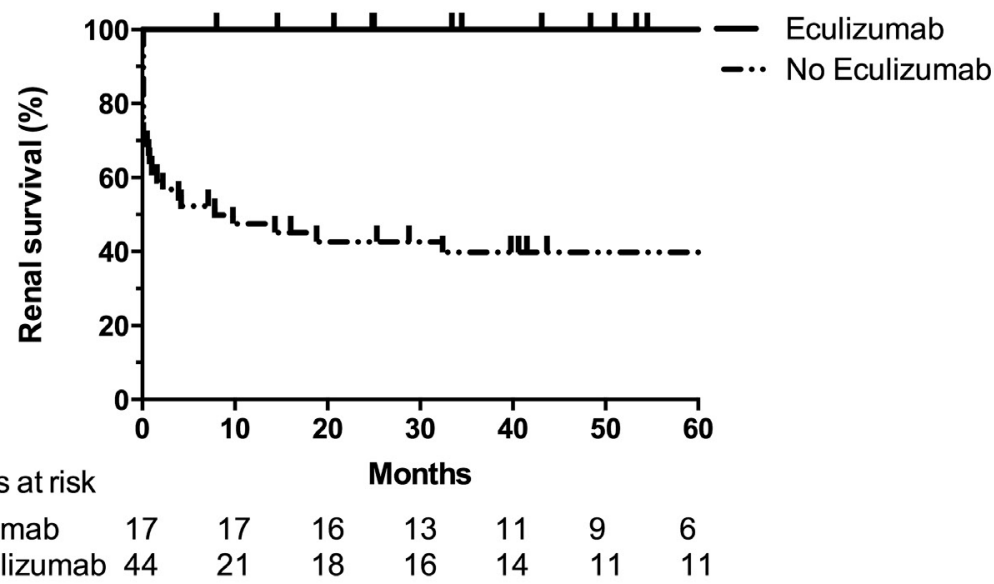

C

Patients at ris

$\begin{array}{lllllllll}\text { Eculizumab } & 30 & 26 & 23 & 18 & 14 & 11 & 7\end{array}$

$\begin{array}{lllllll}\text { No Eculizumab } 105 & 42 & 35 & 30 & 26 & 20 & 18\end{array}$

$\begin{array}{llllllll}\text { Eculizumab } & 17 & 17 & 16 & 13 & 11 & 9 & 6\end{array}$

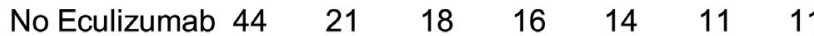

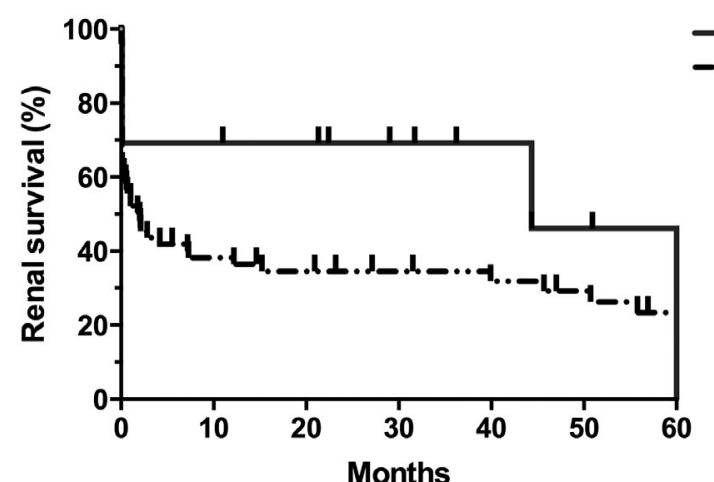

Eculizumab

... No Eculizumab

Patients at risk

$\begin{array}{llllllll}\text { Eculizumab } & 13 & 9 & 9 & 6 & 4 & 3 & 2\end{array}$

Figure 4. Renal survival of patients treated or not with eculizumab. Analysis of renal survival without end-stage renal disease or death according to use of eculizumab treatment in: (A) the whole cohort, $P<0.001$; (B) in patients without hypertensive emergency (HE) and atypical hemolytic uremic syndrome, $P<0.001$; and $(C)$ in HE-aHUS patients, $P=0.18$. Log-rank test. Follow-up was not available for two patients with HE-aHUS. 
Table 3. Characteristics and outcome of patients with atypical hemolytic uremic syndrome according to the presence or absence of hypertensive emergency and rare complement variants.

\section{A. Whole cohort}

\begin{tabular}{|c|c|c|c|c|c|c|}
\hline Categories & HE-aHUS/V & HE-aHUS/noV & noHE-aHUS/V & noHE-aHUS/noV & $P$ & $\begin{array}{c}P \\
\text { HE-aHUS/V } \\
\text { vs. HE-aHUS/noV }\end{array}$ \\
\hline Number & 41 & 35 & 42 & 19 & & \\
\hline Male gender n (\%) & $24(58.5)$ & $20(57.1)$ & $12(28.6)$ & $5(26.3)$ & 0.006 & $>0.9$ \\
\hline Age, mean (SD), years & $40.2(12.19)$ & $33.7(10.11)$ & $34.2(11.35)$ & $41.1(16.8)$ & 0.02 & 0.01 \\
\hline Hemoglobin, mean (SD) g/L & $8.359(1.937)$ & $8.53(2.367)$ & $7.033(1.471)$ & $8.394(1.27)$ & 0.01 & 0.7 \\
\hline Platelets, mean(SD) x109/L & $89.631(46.550)$ & $109.520(38.888)$ & $98.828(56.834)$ & $62.188(45.140)$ & 0.02 & 0.05 \\
\hline Elevated LDH, n (\%) & 20(87) & $14(74)$ & 23(92) & $10(83)$ & 0.4 & 0.4 \\
\hline Presence of schistocytes, n (\%) & 23(82.1) & $18(72)$ & $26(93)$ & $13(87)$ & 0.23 & 0.5 \\
\hline Low C3, n (\%) & $11(20)$ & $1(3)$ & $18(40)$ & $1(0)$ & $<0.0001$ & 0.004 \\
\hline Neurological impairment, n (\%) & $15(65)$ & $17(65)$ & $7(20)$ & $5(28)$ & 0.0002 & $>0.9$ \\
\hline Cardiac dysfunction, n (\%) & $9(24)$ & $7(29)$ & $5(15)$ & $1(6)$ & 0.19 & 0.8 \\
\hline Dialysis at onset, $\mathrm{n}(\%)$ & $33(85)$ & $24(80)$ & $31(82)$ & $10(53)$ & 0.04 & 0.75 \\
\hline
\end{tabular}

B. Patients without hypertensive emergency and without rare complement variant vs. whole cohort excluding those without hypertensive emergency and without rare complement variants.

\begin{tabular}{|c|c|c|c|}
\hline Categories & noHE-aHUS/noV & Whole cohort excluding noHE-aHUS/noV & $\boldsymbol{P}$ \\
\hline Number & 19 & 118 & \\
\hline Male gender, n(\%) & $5(26.3)$ & $56(48)$ & 0.0863 \\
\hline Age, mean (SD), years & $41.1(16.8)$ & $36(11)$ & 0.1065 \\
\hline Hemoglobin, mean (SD) g/L & $8.394(1.27)$ & $8,0(2)$ & 0.490 \\
\hline Platelets, mean (SD) x10\%/L & $62188(45140)$ & 70059 & 0.0391 \\
\hline Elevated LDH, n (\%) & $10(83)$ & $57(85)$ & $>0.9$ \\
\hline Presence of schistocytes, n (\%) & $13(87)$ & $67(82,5)$ & $>0.9$ \\
\hline Low C3, n (\%) & $0(0)$ & $24(21)$ & 0,02 \\
\hline Neurological impairment, n(\%) & $5(28)$ & $39(46)$ & 0.19 \\
\hline Cardiac dysfunction, n (\%) & $1(6)$ & $21(22)$ & 0.19 \\
\hline Dialysis at onset, $\mathrm{n}(\%)$ & $10(53)$ & $88(82)$ & 0.01 \\
\hline
\end{tabular}

HE/V: patients with hypertensive emergency (HE) and atypical hemolytic uremic syndrome (aHUS) with rare complement variants; HE/noV: patients with HE-aHUS without rare complement variants; noHE/V: patients with noHEaHUS with rare complement variants; noHE/noV: patients with noHE-aHUS and no rare complement variants; SD: standard deviation; $\mathrm{LDH}$ : lactate dehydrogenase

\section{Discussion}

Here, we describe the first series of patients with HEaHUS using the new definition of $\mathrm{HE}^{6}$ and compared variant frequencies and clinical outcome in aHUS cases with and without HE. This study showed that a genetic predisposition accounts for half of the patients in both groups and provided data showing that response to treatment and long-term outcome are predicted by HE phenotype at onset and individual gene abnormalities.

In light of the recent advances in the understanding of the pathophysiology of HUS, 2,17 pathogenic variants in complement genes are the hallmark of complementmediated HUS, and have been found in $40-60 \%$ of patients classified as having aHUS. ${ }^{1}$ Currently, there are few data addressing individual susceptibility to aHUS with hypertensive crisis/HE. In this study, we performed extensive complement genetic screening to identify rare variants and at-risk haplotypes identified as the aHUS genetic background. ${ }^{18}$ We identified rare variants in one or two complement genes in $51.3 \%$ of HE-aHUS patients. This frequency is similar to that recently reported in 8/17 patients with 'hypertension-associated thrombotic microangiopathy' and complement variant. ${ }^{12}$ The variant frequency in each gene was not significantly different between HE-aHUS and noHE-aHUS patients. The rare variants identified in noHE-aHUS $(91 \%)$ and in HE-aHUS $(66.6 \%)$ patients lead to quantitative or functional deficiency which impairs the protection of endothelial cells from complement damage and are, therefore, pathogenic. ${ }^{19}$ Interestingly, the frequency of individuals carrying at least one pathogenic variant was significantly higher in HE-aHUS patients than in controls, but significantly lower than in noHE-aHUS patients (control $2.5 \%$, HE-aHUS 39\%, noHE-aHUS 62\%; $P<0.001)$. We also observed a slightly increased frequency of the homozygous CFH at-risk haplotype in HE-aHUS patients compared to that in controls, but did not find any significant difference between controls and noHE-aHUS patients. This observation needs to be confirmed in larger cohorts 
A

Patients at risk

noHE-aHUS without $C$ rare variant 13

noHE-aHUS with $\mathrm{C}$ rare variant

HE-aHUS without $\mathrm{C}$ rare variant

$\mathrm{HE}$-aHUS with $\mathrm{C}$ rare variant

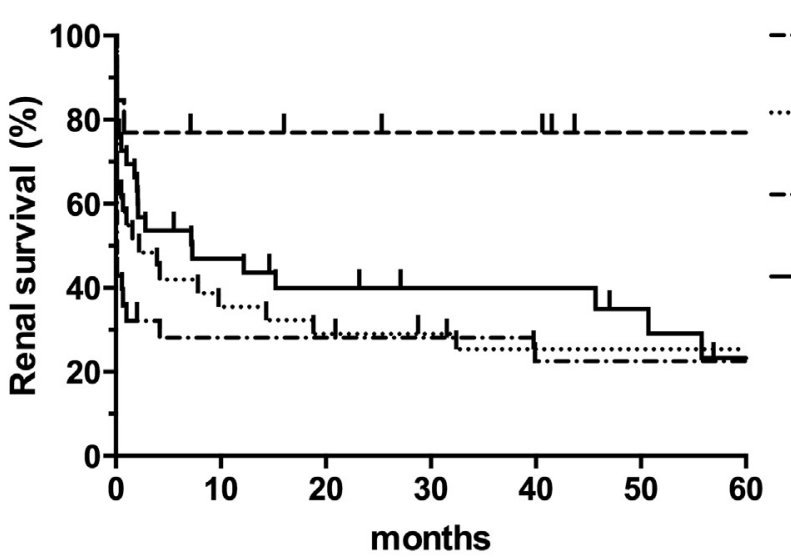

noHE-aHUS without $C$ rare variant

noHE-aHUS with $\mathrm{C}$ rare variant

HE-aHUS without C rare variant

HE-aHUS with C rare variant
B

Patients at risk

noHE-aHUS without $\mathrm{C}$ rare variant 19

noHE-aHUS with $\mathrm{C}$ rare variant 42

HE-aHUS without $C$ rare variant 34

$\mathrm{HE}$-aHUS with $\mathrm{C}$ rare variant

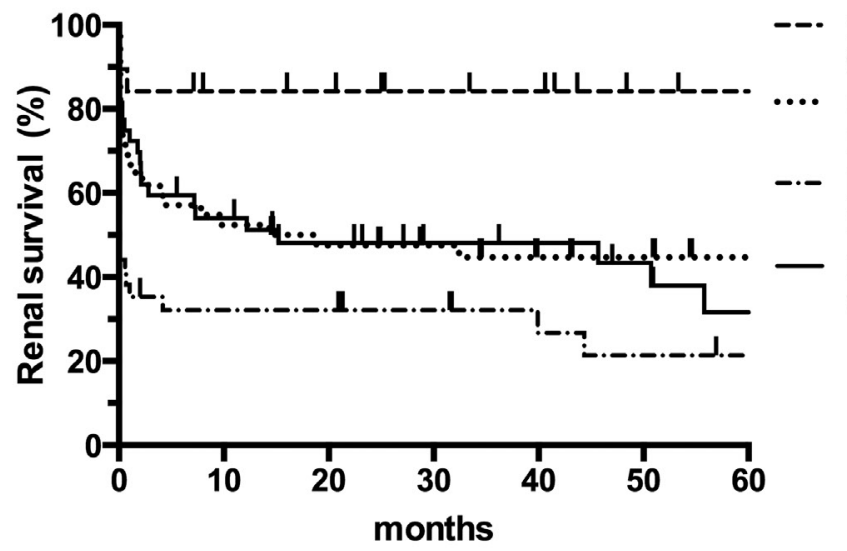

-- noHE-aHUS without $\mathrm{C}$ rare variant noHE-aHUS with $\mathrm{C}$ rare variant

-.- HE-aHUS without C rare variant

HE-aHUS with C rare variant

Figure 5. Renal survival of patients with atypical hemolytic uremic syndrome according to the presence or absence of rare complement variants and hypertensive emergency. (A) Analysis of renal survival without end-stage renal disease or death in patients not treated with eculizumab, log-rank test, $P=0.02$. (B) Analysis of renal survival in the whole cohort, log-rank test, $P=0.001$. Follow-up was not available for two patients with hypertensive emergency and atypical hemolytic uremic syndrome. HE: hypertensive emergency; aHUS: atypical hemolytic uremic syndrome; C: complement.

but may suggest that the $\mathrm{H} 3$ haplotype in the $C F H$ gene confers an increased risk of HUS only in patients with hypertensive crisis. Altogether, we showed that genetically impaired regulation of complement activation is present in a substantial proportion of patients with HEaHUS. However, whether a hypertensive crisis acts as a disease trigger in variant carriers or whether complementmediated endothelium damage induces a secondary HE phenotype remains to be studied. ${ }^{20}$

The cause of the disease remained undetermined in $60 \%$ (46/76) HE-aHUS and 32\% (20/61) of noHE-aHUS patients. Notably, we identified no DGKE mutation in the 76 HE-aHUS patients. Moreover, the absence of effect of complement variants in the renal prognosis of HE-
aHUS patients, contrary to patients with noHE-aHUS, suggests the involvement of superimposed factors other than complement in HE-aHUS. A major involvement of the renin-angiotensin system during HE-aHUS has been demonstrated in the stroke prone spontaneously hypertensive rat model. ${ }^{21}$ Interestingly, C3 is involved in the phenotype of this model, ${ }^{22}$ and renin has been shown to cleave $\mathrm{C} 3$, an effect inhibited by a direct renin inhibitor. ${ }^{23,24}$ Renin-angiotensin system activation is also correlated with hemolysis in HE patients. ${ }^{25}$ Increased chronic renin-angiotensin system activation could have a deleterious role in the long-term kidney prognosis of HEaHUS patients. ${ }^{26}$ Overall, our results suggest that HEaHUS has a complex pathophysiology involving comple- 
ment regulation but also superimposed complementindependent vascular injury.

Interestingly, 12/76 (16\%) HE-aHUS patients presented with a medical history of long-lasting hypertension or left ventricular hypertrophy suggesting previously undiagnosed high blood pressure. Among these 12 patients, seven had rare complement genetic variants. Thus, even in the presence of a history of hypertension, patients with HE-aHUS should be studied for rare complement genetic variants. Among HE-aHUS patients, only slight differences in age and platelet count were observed between those with or without rare complement variants. However, low C3 was significantly more frequent in cases with rare complement variant. Thus, patients with HE-aHUS and low C3 levels should be strongly suspected of having an underlying complement-mediated disease.

Our results showed that a diagnosis of HE-aHUS is relevant from a prognostic standpoint. For the first time, we identified $\mathrm{HE}$ at onset as a new clinical factor which could be associated with long-term prognosis. The renal prognosis was dramatically better in patients without either HE or complement variants than in all other groups, even in the absence of eculizumab treatment. Similarly, a recent study showed that discontinuation of eculizumab after 6-12 months of treatment seems safe in patients with no documented complement variants. ${ }^{27}$ Notably, HE-aHUS patients had a severe renal prognosis even in the absence of a rare complement variant, contrary to that of noHE-aHUS patients, in whom renal prognosis was associated with rare complement variants. Furthermore, the dramatic therapeutic effect of eculizumab in noHEaHUS patients was not observed in HE-aHUS patients, despite a trend to improved prognosis in HE-aHUS patients with a complement variant treated with eculizumab. Overall, our data allow stratification of patients with highly different prognoses according to the presence or absence of $\mathrm{HE}$ or rare complement variants: (i) noHE-aHUS patients without rare complement variants have a favorable prognosis even without eculizumab treatment; (ii) HE-aHUS and noHE-aHUS patients with rare complement variants have a good therapeutic response to eculizumab; and (iii) HE-aHUS patients with no complement variant have a severe prognosis and unclear benefit from eculizumab treatment.

Our study has several limitations. First, our analysis was based on retrospective data. Of greatest concern may be the lack of blood pressure data in $31 \%$ of patients from the aHUS registry, who were therefore excluded from this study. Second, major changes in the management of aHUS occurred during the recruitment period. Only patients diagnosed after 2011 were treated with eculizumab and thus were compared to patients diagnosed before the era of anti-complement therapy. A prospective study is therefore essential in order to evaluate the effectiveness of the treatment. Finally, the scope of the current study did not include cases in which the clinicians considered patients as having HE only or HUS as a direct consequence of high blood pressure. In these cases, clinicians did not perform complement assessment and patients were not enrolled in this study.

In conclusion, our results show that $40 \%$ of patients with HE-aHUS have complement dysregulation with pathogenic rare complement variants. HE and complement genetics allow stratification of patients with highly different renal prognoses and suggest new pathophysiological pathways involved in HUS.

\section{Acknowledgments}

This work was supported by grants from the SFNDT (Société Francophone de Néphrologie, Dialyse et Transplantation) (to KEK), from the Délégation Régionale à la Recherche Clinique, Assistance Publique - Hôpitaux de Paris (Programme Hospitalier de Recherche Clinique (AOM08198) (to VFB), by the EU FP7 grant 2012-305608 (EURenOmics) (to VFB), the Fondation Du Rein (FRM, Prix 2012 FDR) (to VFB), and the Association pour l'Information et la Recherche dans les Maladies Rénales Génétiques (AIRG France). FP is supported by a CARPEM (CAncer Research for PErsonalized Medicine) doctoral fellowship.

\section{References}

1. Fakhouri F, Zuber J, Fremeaux-Bacchi V, Loirat C. Haemolytic uraemic syndrome. Lancet. 2017;390(10095):681-696.

2. Jokiranta TS. HUS and atypical HUS. Blood. 2017;129(21):2847-2856.

3. Masias C, Vasu S, Cataland SR. None of the above: thrombotic microangiopathy beyond TTP and HUS. Blood. 2017;129(21):28572863.

4. Legendre CM, Licht C, Muus $\mathrm{P}$, et al. Terminal complement inhibitor eculizumab in atypical hemolytic-uremic syndrome. N Engl J Med. 2013;368(23):2169-2181.

5. Cremer A, Amraoui F, Lip GY, et al. From malignant hypertension to hypertensionMOD: a modern definition for an old but still dangerous emergency. J Hum Hypertens. 2016;30(8):463-466.

6. Mancia G, Fagard R, Narkiewicz K, et al. 2013 ESH/ESC guidelines for the manage- ment of arterial hypertension: the Task Force for the Management of Arterial Hypertension of the European Society of Hypertension (ESH) and of the European Society of Cardiology (ESC). Eur Heart J. 2013;34(28):2159-2219

7. van den Born BJ, Honnebier UP, Koopmans $\mathrm{RP}$, van Montfrans GA. Microangiopathic hemolysis and renal failure in malignant hypertension. Hypertension. 2005;45(2): 246-251.

8. van den Born BJ, van der Hoeven NV, Groot $\mathrm{E}$, et al. Association between thrombotic microangiopathy and reduced ADAMTS13 activity in malignant hypertension. Hypertension. 2008;51(4):862-866.

9. Fremeaux-Bacchi V, Fakhouri F, Garnier A, et al. Genetics and outcome of atypical hemolytic uremic syndrome: a nationwide French series comparing children and adults. Clin J Am Soc Nephrol. 2013;8(4):554-562.

10. Larsen CP, Wilson JD, Best-Rocha A, Beggs
ML, Hennigar RA. Genetic testing of complement and coagulation pathways in patients with severe hypertension and renal microangiopathy. Mod Pathol. 2018;31(3): 488-494.

11. Noris M, Caprioli J, Bresin E, et al. Relative role of genetic complement abnormalities in sporadic and familial aHUS and their impact on clinical phenotype. Clin J Am Soc Nephrol. 2010;5(10):1844-1859.

12. Timmermans S, Abdul-Hamid MA, Potjewijd J et al. C5b9 formation on endothelial cells reflects complement defects among patients with renal thrombotic microangiopathy and severe hypertension. J Am Soc Nephrol. 2018;29 (8):2234-2243

13. Vieira-Martins P, El Sissy C, Bordereau P, Gruber A, Rosain J, Fremeaux-Bacchi V. Defining the genetics of thrombotic microangiopathies. Transfus Apher Sci. 2016;54(2):212-219. 
14. Richards S, Aziz N, Bale S, et al. Standards and guidelines for the interpretation of sequence variants: a joint consensus recommendation of the American College of Medical Genetics and Genomics and the Association for Molecular Pathology. Genet Med. 2015;17(5):405-424

15. Goodship TH, Cook HT, Fakhouri F, et al. Atypical hemolytic uremic syndrome and C3 glomerulopathy: conclusions from a "Kidney Disease: Improving Global Outcomes" (KDIGO) Controversies Conference. Kidney Int. 2017;91(3):539-551.

16. Foster MC, Coresh J, Fornage $M$, et al. APOL1 variants associate with increased risk of CKD among African Americans. J Am Soc Nephrol. 2013;24(9):1484-1491.

17. Kremer Hovinga JA, Heeb SR, Skowronska M, Schaller M. Pathophysiology of thrombotic thrombocytopenic purpura and hemolytic uremic syndrome. I Thromb Haemost. 2018;16(4):618-629.

18. Fremeaux-Bacchi V, Kemp EJ, Goodship JA, et al. The development of atypical haemolytic-uraemic syndrome is influenced by susceptibility factors in factor $\mathrm{H}$ and membrane cofactor protein: evidence from two independent cohorts. J Med Genet. 2005;42(11):852-856.

19. Osborne AJ, Breno M, Borsa NG, et al. Statistical validation of rare complement variants provides insights into the molecular basis of atypical hemolytic uremic syndrome and C3 glomerulopathy. J Immunol. 2018;200(7):2464-2478.

20. Mathew RO, Nayer A, Asif A. The endothelium as the common denominator in malignant hypertension and thrombotic microangiopathy. JASH. 2016;10(4):352-359.

21. Chander PN, Rocha R, Ranaudo J, Singh G, Zuckerman A, Stier CT, Jr. Aldosterone plays a pivotal role in the pathogenesis of thrombotic microangiopathy in SHRSP. Am Soc Nephrol. 2003;14(8):1990-1997.

22. Negishi E, Fukuda N, Otsuki T, et al. Involvement of complement 3 in the saltsensitive hypertension by activation of renal renin-angiotensin system in spontaneously hypertensive rats. Am J Physiol Renal Physiol. 2018;315(6):F1747-f1758.
23. Bekassy ZD, Kristoffersson AC, Rebetz J, Tati R, Olin AI, Karpman D. Aliskiren inhibits renin-mediated complement activation. Kidney int. 2018;94(4):689-700.

24. Raghunathan V, Sethi SK, Dragon-Durey $\mathrm{MA}$, et al. Targeting renin-angiotensin system in malignant hypertension in atypical hemolytic uremic syndrome. Indian J Nephrol. 2017;27(2):136-140.

25. van den Born BJ, Koopmans RP, van Montfrans GA. The renin-angiotensin system in malignant hypertension revisited: plasma renin activity, microangiopathic hemolysis, and renal failure in malignant hypertension. Am J Hypertens. 2007;20 (8):900-906

26. Romagnani P, Remuzzi G, Glassock R, et al Chronic kidney disease. Nat Rev Dis Primers. 2017;3:17088.

27. Fakhouri F, Fila M, Provot F, et al Pathogenic variants in complement genes and risk of atypical hemolytic uremic syndrome relapse after eculizumab discontinuation. Clin J Am Soc Nephrol. 2017;12(1) :50-59. 